Original Contribution

\title{
ANALYSIS OF STABILITY AND ADAPTATION OF COTTON GENOTYPES USING GGE BIPLOT METHOD
}

\author{
M. Fathi Sadabadi ${ }^{1,2 *}$, G. A. Ranjbar ${ }^{1}$, M. R. Zangi², S. K. Kazemi Tabar ${ }^{1}$, \\ H. Najafi Zarini ${ }^{1}$ \\ ${ }^{1}$ Department of Agronomy and Plant Breeding, Sari university of Agricultural Science and Natural \\ Resource, Iran \\ ${ }^{2}$ Cotton Research Institute of Iran, Agricultural Research, Education and Extension Organization \\ (AREEO), Gorgan, Iran.
}

\begin{abstract}
This research was conducted to study effects of $\mathrm{G} \times \mathrm{E}$ interaction on 38 selected genotypes of cotton with two commercial cultivars Golestan and Sepid (control) in a randomized complete block design with three replications at three locations in Golestan Province in 2014-15. The measured characteristics were included: plant height, sympodial length, sympodial number, boll number, boll weight, seed cotton yield and earliness. Analysis of variance showed that genotype effect is significant in 1 or $5 \%$ probability levels on measured traits except for boll number and earliness. A significant interaction effect between genotype $\times$ locations in yield showed different variation trends in various locations. So that genotype 29 had the best performance in Hashemabad station but genotypes 24 and 18 showed their best performances in Gonbad and Anbarolum station respectively. Stability parameters were calculated and genotype No. 8 was defined according to regression slope close to 1 as the most stable genotypes among. This genotype had a smaller share in genotype and environment interaction according to Rick ecovalans and Shukla stability variance parameters and 10 and 33 were the most unstable genotypes in terms of performance. GGE biplot method showed that the first two principal components regression model explained $74 \%$ of the observed changes. GGE biplot graph plotted by software reflected the superior genotypes TJ82, ER26, DB29, DB19, DB25 and ER36 respectively. Also Hashemabad has been identified as appropriate region for ER26 genotype and TJ82 was identified as the best and most stable genotype.
\end{abstract}

Keywords: genotype and environment interaction, GGE Biplot, Cotton, Genotype, stability, adaptability.

\section{INTRODUCTION}

Cotton (Gossypium sp. L.) as a tropical and subtropical plant is grown worldwide. Much of the cotton crop is produced in Asia, as four Asian countries China, India, Pakistan and Uzbekistan produce about $68 \%$ of world cotton, however, USA would alone produces about $12 \%$ of the world's cotton. The role of cotton in gross domestic product (GDP) of Pakistan is $1.6 \%$ and in Syria $2.5 \%$. The average yield in crop year 2009 to 2008 in China, India, Uzbekistan and Pakistan, are 3963, 1569, 2115 and $1959 \mathrm{~kg}$ per hectare, respectively (1). Cotton cultivation area in Iran is reported about 101 thousands hectares

\footnotetext{
*Correspondence to: Mohsen Fathi Sadabadi (M.Sc), Dept. of Cotton Breeding, Cotton Research Institute (CRI), Shahid Beheshti Aven. Zip Code, 49166-85915, Gorgan, IRAN, Tel:+989113756573, Fax:+981732227781,mohsenfathi433@yahoo.com
}

in 2012-2013 that in comparison with the past decade, approximately 40 to 50 percent of the cultivated area has been reduced (2).

Introducing lines with high and stable yield for different regions with diverse climatic conditions is one way of increasing crop production. For selection and introduction of high yielding and stable varieties, replicated yield trials in a number of years and places are conducted. In these experiments, usually after the decomposition of variance when interaction between genotype and environment is existed, stability of genotypes in addition to performance criteria should also be considered for introducing new variety (3).

Alishah et al. (2) investigated 9 new varieties of cotton's agronomic characteristics in comparison with commercial varieties, Varamin, Sahel and Bakhtegan, in 9 regions 
during 2008-2009. Based on results of year $\times$ location interaction for all quality and earliness, yield and boll weight were significantly differed at $1 \%$ probability level. Interaction of year $\times$ genotype for fiber quality and boll weight was not significant. All cultivars had average stability to different environments, and among them cultivars SP732, SP731 and NNB had good compatibility, besides hybrid NNC as new varieties recommended for cotton areas of the country have been introduced. Damavandi et al. (4) investigated genotype $\times$ environment interactions and stability of performance of 10 cotton varieties in six regions through parametric univariate method, non-parametric and AMMI model and found that $81 \%$ of changes have been occurred by two main factors I and II (IPC1,2). Based on biplot diagram of components of genotype and environment and average yield of genotypes in different environments, Chekurova, Sepid and Tabladila were the most stable cultivars, however, the cultivars 43200, Khordad and Sahel showed the highest $\mathrm{G} \times \mathrm{E}$ interaction. Meanwhile, Pourdad and Jamshidy Moghadam (5) using GGE Biplot to study grain yield of 9 brasica napus genotypes in four regions over two years and their research led to identify 6 superior genotypes and two big environments. Also proper genotypes were determined within each environment.

Lalbachan (6) reported significant genotype and environment interaction for grain yield in rice and defined the stable genotypes using Eberhart and Russell method. Moreover, Li et al. (7) in stability analysis of important economic traits reported 7 cultivars in 12 districts in 3 years, 4 digits K326, K346, Yuniyan87 and RJ17 in terms of economic characteristics were better than the others. However, both K315 and K358 in yield and average price in areas have proper stability.

Campbell \& Jones (8) evaluated genotype $\times$ environment interactions effects on agronomic performance (lint yield, gin turnout) and fiber quality (fiber length, fiber strength, uniformity index, micronaire, fiber elongation) in a series of cotton (Gossypium hirsutum) performance trials in 12 location-year environments in South Carolina. Result showed genotype $\times$ environment interactions affecting lint yield were larger in higher yielding environments, while interactions for fiber strength were greater for genotypes with lower mean fiber strength values.

Farshadfar \& et al. (9) In order to determine stable bread wheat genotypes with high grain yield via a single parameter, field experiments were conducted with 14 genotypes for 3 consecutive years (2008-2011) under two different conditions (irrigated and rain fed) in a complete randomized block design with three replications in each environment. Combined analysis of variance showed highly significant differences for the GE (genotype-environment) interaction indicating the possibility of selection for stable entries. The results of AMMI (additive main effect and multiplicative interaction) analysis indicated that the first four AMMI (AMMI1-AMMI4) were highly significant $(\mathrm{P}<0.01)$.

In present studied, a group of superior genotypes were tested at several environments (stress of saline and non-saline) in terms of quantity and quality characters.

\section{MATERIALS AND METHODS}

Thirty eight new cotton genotypes along with two commercial cultivars of Golestan and Sepid (control) were evaluated in a randomized complete block design with three replications in three regions (Hashemabad, Anbarolum and Gonbad) in Golestan province during 2014-2015. Field has been usually prepared and cotton crop technical guideline was carried out. Winter and spring cultivators to provide suitable planting was done in due time. Planting has been performed using $20 \times 80 \mathrm{~cm}$ pattern in second half of May in each year. To provide enough green area, thinning and weeding operations were performed during the growing season. Urea, ammonium phosphate and potassium sulphate fertilizers were applied as recommendation based on the soil sample analysis.

The measured characteristics were included: plant height, sympodial length, sympodial number, boll number, boll weight, seed cotton yield and earliness (the proportion of cotton seed of the first harvest on total performance). In order to measure the characteristics of each treatment, five random plants have been randomly selected, and all data recorded only from the selected plants. Harvesting was done after removing the half a meter from up and down of rows. For data analysis, and to evaluate the stability of genotypes and drawing graphs, SAS 9.0 and GGE biplot software were used, respectively.

\section{RESULTS AND DISCUSSION}

Analysis of variance showed that there are significant differences at 5 or $1 \%$ level among the studied genotypes in years and locations in most of traits. However, genotype $\times$ location interaction was significant for length of sympodial and yield. It is suggested that for these traits there are various process of variation in different locations. So that, a single genotype cannot be introduced for planting in all areas, instead, it is necessary to recommend a proper genotype for each distinct region (Table 1). 
SADABADI M. FATHI, et al.

Table 1. Combined analysis of variance cotton genotypes in three regions Hashemabad, Anbarolum and Gonbad (2014-15)

\begin{tabular}{|c|c|c|c|c|c|c|c|c|}
\hline $\begin{array}{l}\text { Source of } \\
\text { variation }\end{array}$ & $\mathrm{df}$ & earliness & $\begin{array}{c}\text { Cotton } \\
\text { seed }\end{array}$ & $\begin{array}{c}\text { Boll } \\
\text { weight }\end{array}$ & $\begin{array}{c}\text { Boll } \\
\text { number }\end{array}$ & $\begin{array}{c}\text { Sympodial } \\
\text { number }\end{array}$ & $\begin{array}{l}\text { Sympodial } \\
\text { length }\end{array}$ & $\begin{array}{l}\text { Plant } \\
\text { high }\end{array}$ \\
\hline & & \multicolumn{7}{|c|}{ Mean of square } \\
\hline Year $(\mathrm{Y})$ & 1 & $101.4^{\mathrm{ns}}$ & $120228883.9^{* * *}$ & $192.1^{* * *}$ & $1200.8^{* * *}$ & $399.6^{* *}$ & $1224.3^{* *}$ & $21974.3^{* * *}$ \\
\hline Location (L) & 2 & $147485.7^{* *}$ & $206486301.9^{* *}$ & $310.4^{* *}$ & $5212.6^{* *}$ & $2756.5^{* *}$ & $8560.5^{* *}$ & $122493.3^{*}$ \\
\hline $\mathrm{Y} * \mathrm{~L}$ & 2 & $25586.1^{* *}$ & $6643108.7^{* *}$ & $20.2^{* *}$ & $10.3^{\mathrm{ns}}$ & $562.7^{* *}$ & $1037.7^{* *}$ & $17083.4^{* *}$ \\
\hline $\operatorname{Rep}\left(\mathrm{Y}^{*} \mathrm{~L}\right)$ & 12 & 33234.5 & 2975776.6 & 5.69 & 46.7 & 114.9 & 278.2 & 5220.7 \\
\hline Genotype & 39 & $81432.1^{* * *}$ & $1122640.2^{* * *}$ & $2.09^{* *}$ & $15.6^{*}$ & $26.9^{* *}$ & $97.7^{* *}$ & $1537.4^{* *}$ \\
\hline $\mathrm{Y}^{*} \mathrm{G}$ & 39 & $19467.2^{* *}$ & $311302.3^{\mathrm{ns}}$ & $1.06^{*}$ & $9.15^{\mathrm{ns}}$ & $11.1^{\mathrm{ns}}$ & $42.1^{\mathrm{ns}}$ & $323.4^{\mathrm{ns}}$ \\
\hline $\mathrm{L} * \mathrm{G}$ & 78 & $34752.6^{\mathrm{ns}}$ & $518293.7^{* *}$ & $1.73^{* *}$ & $12.8^{\mathrm{ns}}$ & $15.1^{*}$ & $84.1^{* *}$ & $557.8^{*}$ \\
\hline $\mathrm{Y} * \mathrm{~L} * \mathrm{G}$ & 78 & $47404.7^{* *}$ & $227726.1^{\mathrm{ns}}$ & $0.817^{\mathrm{ns}}$ & $9.41^{\mathrm{ns}}$ & $5.19^{\mathrm{ns}}$ & $36.1^{\mathrm{ns}}$ & $259.3^{\text {ns }}$ \\
\hline error & 468 & 190860.8 & 306914.6 & 0.759 & 10.2 & 10.4 & 43.9 & 405.3 \\
\hline C.V & & 19.5 & 22.3 & 22.1 & 22.9 & 28.3 & 26.5 & 26.5 \\
\hline
\end{tabular}

ns: not significant differences $*$ : significant at the $5 \%$ level $* *$ significant at the $1 \%$ level.

Figure 1 shows the trends of genotypes performance in different regions. Genotypes 29,24 and 18 showed the best performance in
Hashemabad, Anbarolum and Gonbad, respectively.

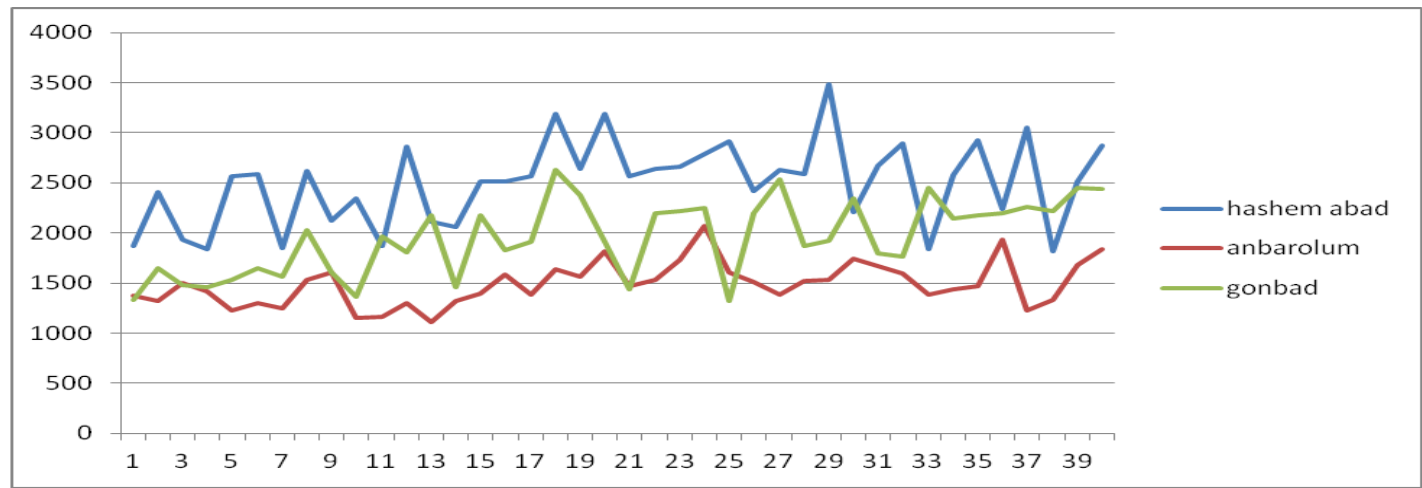

Figure 1. Performance changes trends in cotton genotypes in different areas

According to the results (Table 2) the first year was superior to the second year in most of the studied traits. Meanwhile, among regions of running area, Hashemabad, Gonbad and Anbarolum are placed from first to third for nearly all measured traits, respectively.

Table 2. Comparing the average trait in the years and different areas of the project

\begin{tabular}{ccccccccc}
\hline & & Earliness & $\begin{array}{c}\text { Seed } \\
\text { Cotton } \\
(\mathrm{Kg} / \mathrm{ha})\end{array}$ & $\begin{array}{c}\text { Boll } \\
\text { Weight } \\
(\mathrm{gr})\end{array}$ & $\begin{array}{c}\text { Boll } \\
\text { Number }\end{array}$ & $\begin{array}{c}\text { Sympodial } \\
\text { Number }\end{array}$ & $\begin{array}{c}\text { Sympodial } \\
\text { Length } \\
(\mathrm{cm})\end{array}$ & $\begin{array}{c}\text { Plant } \\
\text { High } \\
(\mathrm{cm})\end{array}$ \\
\hline year & 2014 & $69.1 \mathrm{a}$ & $2122 \mathrm{a}$ & $4.44 \mathrm{a}$ & $8.87 \mathrm{a}$ & $12.1 \mathrm{a}$ & $19.4 \mathrm{a}$ & $82.7 \mathrm{a}$ \\
& 2015 & $69.8 \mathrm{a}$ & $1705 \mathrm{~b}$ & $3.41 \mathrm{~b}$ & $6.28 \mathrm{~b}$ & $10.6 \mathrm{~b}$ & $16.8 \mathrm{~b}$ & $71.7 \mathrm{~b}$ \\
\hline Location & Hashem- & $79.7 \mathrm{a}$ & $2498 \mathrm{a}$ & $4.83 \mathrm{a}$ & $12.9 \mathrm{a}$ & $15.2 \mathrm{a}$ & $23.5 \mathrm{a}$ & $97.2 \mathrm{a}$ \\
& Abad & & & & & & & \\
& Anbarolum & $49.2 \mathrm{~b}$ & $1089 \mathrm{c}$ & $2.65 \mathrm{c}$ & $4.53 \mathrm{~b}$ & $8.6 \mathrm{c}$ & $11.7 \mathrm{c}$ & $52.7 \mathrm{c}$ \\
& Gonbad & $79.5 \mathrm{a}$ & $1954 \mathrm{~b}$ & $4.31 \mathrm{~b}$ & $5.26 \mathrm{~b}$ & $10.4 \mathrm{~b}$ & $18.9 \mathrm{~b}$ & $81.7 \mathrm{~b}$ \\
\hline Interaction & $\mathrm{Y} 1 * \mathrm{~L} 1$ & $86.4 \mathrm{a}$ & $3014 \mathrm{a}$ & $5.08 \mathrm{a}$ & $14.1 \mathrm{a}$ & $14.2 \mathrm{~b}$ & 23.0 & $97.3 \mathrm{a}$ \\
Year*Location & & & & & & & & \\
& $\mathrm{Y} 1 * \mathrm{~L} 2$ & $41.4 \mathrm{e}$ & $1183 \mathrm{e}$ & $3.48 \mathrm{~d}$ & $5.7 \mathrm{~d}$ & $10.5 \mathrm{~d}$ & $15.3 \mathrm{~d}$ & $79.0 \mathrm{c}$ \\
& $\mathrm{Y} 1 * \mathrm{~L} 3$ & $79.4 \mathrm{~b}$ & $2170 \mathrm{~b}$ & $4.78 \mathrm{~b}$ & $6.8 \mathrm{c}$ & $11.7 \mathrm{c}$ & $20.0 \mathrm{~b}$ & $83.0 \mathrm{~b}$ \\
& $\mathrm{Y} 2 * \mathrm{~L} 1$ & $73.0 \mathrm{c}$ & $1982 \mathrm{c}$ & $4.59 \mathrm{~b}$ & $11.8 \mathrm{~b}$ & $16.2 \mathrm{a}$ & $24.2 \mathrm{a}$ & $97.1 \mathrm{a}$ \\
& $\mathrm{Y} 2 * \mathrm{~L} 2$ & $57.1 \mathrm{~d}$ & $1197 \mathrm{f}$ & $2.83 \mathrm{e}$ & $3.4 \mathrm{e}$ & $6.7 \mathrm{f}$ & $8.2 \mathrm{e}$ & $37.5 \mathrm{~d}$ \\
& $\mathrm{Y} 2 * \mathrm{~L} 3$ & $79.5 \mathrm{~b}$ & $1736 \mathrm{~d}$ & $3.83 \mathrm{c}$ & $3.7 \mathrm{e}$ & $9.1 \mathrm{e}$ & $18.0 \mathrm{c}$ & $80.5 \mathrm{~b}$ \\
\hline
\end{tabular}

Comparison of average traits in different genotypes was performed using Duncan method in level $1 \%$ (Table 3). The tallest plants were belonged to genotype 25 with a height more than $101 \mathrm{~cm}$ and the shortest plants were belonged to genotype 33, with an average of $64 \mathrm{~cm}$. Maximum length and number of sympodial branches was belonged to genotypes 5 and 24, respectively. This morphological characteristic in cotton is very important because of directly 
SADABADI M. FATHI, et al.

formation of bolls on fruiting branches. Thus, sympodial branches, as components of the performance, can have a positive role in the formation of bolls and increasing yield of cotton.

The highest number of bolls per plant is belonged to the treatment 18 . The number of boll in cotton is so important because the cotton plant has a self-regulating mode and in stress conditions can retain the portion of leaf to time by falling flower and boll. In the contrary, if the conditions are provided by increasing the number of bolls per plant, the maximum performance will produce perhaps why treatment 18 has had the most yields among 40 genotypes studied.
In Golestan, the highest produced yield was harvested in first cutting. But overall the yield harvested, the treatment was number 18 that was superior to all treatments and belonged the highest average yield to $2220 \mathrm{~kg}$ per hectare. The most important point is that the most performance of single plant was produced with this figure of 50 grams per plant. In terms of earliness that in cotton is calculated from the portion of first cutting to total performance, treatment 39 was earlier than others, because 87 percent of the performance of these genotypes was harvested in the first cutting (Table 3).

Table 3. Comparison of mean in the three regions Hashemabad, Anbarolum and Gonbad

\begin{tabular}{|c|c|c|c|c|c|c|c|c|}
\hline Treatment & Genotype & $\begin{array}{c}\text { Earliness } \\
(\%)\end{array}$ & $\begin{array}{c}\text { Seed } \\
\text { Cotton } \\
(\mathrm{Kg} / \mathrm{ha})\end{array}$ & $\begin{array}{c}\text { Boll } \\
\text { Weight } \\
\text { (gr) }\end{array}$ & $\begin{array}{c}\text { Boll } \\
\text { Number }\end{array}$ & $\begin{array}{c}\text { Sympodial } \\
\text { Number }\end{array}$ & $\begin{array}{l}\text { Sympodial } \\
\text { Length } \\
(\mathrm{cm})\end{array}$ & $\begin{array}{l}\text { Plant } \\
\text { High } \\
(\mathrm{cm})\end{array}$ \\
\hline 1 & D39 & $48 \mathrm{jkl}$ & $1256 \mathrm{e}$ & $3.54 \mathrm{e}-\mathrm{i}$ & $6.53 \mathrm{edf}$ & $9.2 \mathrm{fg}$ & $17.4 \mathrm{a}-\mathrm{i}$ & 67.2efg \\
\hline 2 & T52 & $66 b-i$ & $1523 \mathrm{c}-\mathrm{e}$ & $4.05 \mathrm{a}-\mathrm{i}$ & $8.45 \mathrm{a}-\mathrm{e}$ & $11.8 \mathrm{a}-\mathrm{g}$ & $17.8 \mathrm{a}-\mathrm{i}$ & $82.8 b-f$ \\
\hline 3 & D38 & 71a-h & 1367de & 4.09a-f & $6.84 b-f$ & $10.3 \mathrm{~d}-\mathrm{g}$ & $17.1 b-\mathrm{i}$ & 67.6efg \\
\hline 4 & D8 & $62 d-k$ & $1304 d e$ & $4.66 \mathrm{a}$ & $6.44 \mathrm{edf}$ & $11.4 \mathrm{a}-\mathrm{g}$ & $17.4 \mathrm{a}-\mathrm{i}$ & 68.7efg \\
\hline 5 & D26 & $52 \mathrm{i}-1$ & $1509 c-e$ & $3.74 c-j$ & $8.03 \mathrm{a}-\mathrm{f}$ & $10.8 b-g$ & $22.9 \mathrm{a}$ & $70.6 \mathrm{~d}-\mathrm{g}$ \\
\hline 6 & D37 & $60 \mathrm{e}-\mathrm{k}$ & $1578 \mathrm{e}$ & $3.85 \mathrm{~b}-\mathrm{j}$ & 7.30a-f & $9.5 \mathrm{efg}$ & $18.5 \mathrm{a}-\mathrm{g}$ & $67.7 \mathrm{efg}$ \\
\hline 7 & D7 & $55 \mathrm{~h}-\mathrm{k}$ & $1285 b-e$ & $4.01 \mathrm{a}-\mathrm{i}$ & 7.19a-f & $10.1 \mathrm{~d}-\mathrm{g}$ & $15.2 \mathrm{f}-\mathrm{i}$ & $66.4 \mathrm{ef}$ \\
\hline 8 & D9 & $59 \mathrm{f}-\mathrm{k}$ & $1794 b-e$ & 4.20a-f & $7.68 \mathrm{a}-\mathrm{f}$ & $11.6 \mathrm{a}-\mathrm{g}$ & $16.6 \mathrm{~d}-\mathrm{i}$ & $87.8 \mathrm{a}-\mathrm{d}$ \\
\hline 9 & D13 & $64 c-j$ & $1511 \mathrm{c}-\mathrm{e}$ & $4.33 \mathrm{a}-\mathrm{d}$ & $6.92 \mathrm{a}-\mathrm{f}$ & $10.8 \mathrm{~b}-\mathrm{g}$ & $18.5 \mathrm{a}-\mathrm{g}$ & $75.5 \mathrm{c}-\mathrm{g}$ \\
\hline 10 & D25 & 421 & $1355 \mathrm{de}$ & $3.01 \mathrm{j}$ & $6.96 a-f$ & $9.1 \mathrm{~g}$ & $18.3 \mathrm{a}-\mathrm{i}$ & $66.3 \mathrm{ef}$ \\
\hline 11 & T51 & $58 \mathrm{~g}-\mathrm{k}$ & 1396de & $4.01 \mathrm{a}-\mathrm{i}$ & 7.98a-f & $12.5 \mathrm{a}-\mathrm{d}$ & $22.4 \mathrm{ab}$ & $79.1 \mathrm{c}-\mathrm{g}$ \\
\hline 12 & $\mathrm{~T} 41$ & 68b-h & $1721 b-e$ & $4.51 \mathrm{ab}$ & $6.69 c-f$ & $13.5 \mathrm{ab}$ & 21.9abc & $90.4 \mathrm{abc}$ \\
\hline 13 & D10 & $54 \mathrm{~h}-\mathrm{k}$ & $1532 \mathrm{c}-\mathrm{e}$ & $3.98 \mathrm{a}-\mathrm{i}$ & $5.51 \mathrm{f}$ & 9.7efg & $19.1 \mathrm{a}-\mathrm{g}$ & $67.4 \mathrm{efg}$ \\
\hline 14 & D36 & $46 \mathrm{kl}$ & $1347 \mathrm{de}$ & $3.82 \mathrm{~b}-\mathrm{j}$ & $6.96 a-f$ & $11.2 \mathrm{a}-\mathrm{g}$ & $20.7 a-f$ & $80.8 b-g$ \\
\hline 15 & TJ8 & $79 a-d$ & $1759 \mathrm{~b}-\mathrm{e}$ & $4.05 a-i$ & 7.01a-f & $10.3 \mathrm{~d}-\mathrm{g}$ & $17.1 \mathrm{~b}-\mathrm{i}$ & $74.4 \mathrm{c}-\mathrm{g}$ \\
\hline 16 & TJ20 & $71 \mathrm{a}-\mathrm{h}$ & $1708 b-e$ & $3.49 \mathrm{f}-\mathrm{i}$ & $7.28 \mathrm{a}-\mathrm{f}$ & $10.7 \mathrm{c}-\mathrm{g}$ & $16.2 \mathrm{~d}-\mathrm{i}$ & $75.7 \mathrm{c}-\mathrm{e}$ \\
\hline 17 & TJ57 & $68 b-i$ & $1688 b-e$ & $3.77 b-j$ & 7.03a-f & $10.8 \mathrm{~b}-\mathrm{g}$ & $16.5 \mathrm{~d}-\mathrm{i}$ & 67.9efg \\
\hline 18 & TJ82 & $76 a-f$ & $2220 \mathrm{a}$ & $4.27 \mathrm{a}-\mathrm{e}$ & $9.72 \mathrm{a}$ & 13.1abc & 20.6a-f & $91.1 \mathrm{abc}$ \\
\hline 19 & TJ124 & $79 a-d$ & $1942 a-c$ & $3.93 \mathrm{a}-\mathrm{i}$ & $8.02 \mathrm{a}-\mathrm{f}$ & $12.2 \mathrm{a}-\mathrm{e}$ & $16.3 \mathrm{~d}-\mathrm{i}$ & $87.2 \mathrm{a}-\mathrm{d}$ \\
\hline 20 & TJ135 & $66 b-i$ & $2040 \mathrm{ab}$ & $3.92 \mathrm{a}-\mathrm{i}$ & 9.19abc & $11.5 \mathrm{a}-\mathrm{g}$ & $21.6 a-d$ & 84.1a-e \\
\hline 21 & TJ139 & $72 \mathrm{a}-\mathrm{g}$ & $1555 \mathrm{c}-\mathrm{e}$ & $3.87 \mathrm{~b}-\mathrm{i}$ & 7.18a-f & $12.3 \mathrm{a}-\mathrm{e}$ & 20.9a-e & $85.8 \mathrm{a}-\mathrm{d}$ \\
\hline 22 & TJ168 & $68 b-i$ & $1857 \mathrm{~b}-\mathrm{e}$ & $4.25 \mathrm{a}-\mathrm{e}$ & $7.53 \mathrm{a}-\mathrm{f}$ & $10.6 \mathrm{c}-\mathrm{g}$ & $16.6 \mathrm{~d}-\mathrm{i}$ & $77.8 \mathrm{c}-\mathrm{g}$ \\
\hline 23 & TJ169 & $75 a-g$ & $1938 a-c$ & $3.37 \mathrm{c}-\mathrm{j}$ & $7.18 \mathrm{a}-\mathrm{f}$ & $12.8 \mathrm{a}-\mathrm{d}$ & $17.3 \mathrm{a}-\mathrm{i}$ & 88.9abc \\
\hline 24 & TJ174 & $77 a-e$ & $2103 \mathrm{ab}$ & $4.09 a-f$ & 7.31a-f & $13.9 \mathrm{a}$ & $17.2 \mathrm{~b}-\mathrm{i}$ & $97.1 \mathrm{ab}$ \\
\hline 25 & TJ178 & $70 \mathrm{a}-\mathrm{g}$ & $1680 b-e$ & $4.23 \mathrm{a}-\mathrm{e}$ & 8.41a-e & 13.1abc & 19.6a-h & $101.3 \mathrm{a}$ \\
\hline 26 & TJ183 & $73 a-g$ & $1774 b-e$ & $3.63 \mathrm{~d}-\mathrm{j}$ & $7.34 \mathrm{a}-\mathrm{f}$ & $11.1 \mathrm{~b}-\mathrm{g}$ & $19.4 \mathrm{a}-\mathrm{g}$ & $83.3 b-f$ \\
\hline 27 & TJ185 & $74 a-g$ & $1914 a-d$ & $3.93 \mathrm{a}-\mathrm{i}$ & $8.52 \mathrm{a}-\mathrm{d}$ & $12.3 \mathrm{a}-\mathrm{e}$ & 20.3a-f & $84.4 \mathrm{a}-\mathrm{e}$ \\
\hline 28 & TJ189 & $77 a-e$ & $1727 b-e$ & 4.47abc & $6.16 \mathrm{ef}$ & $10.6 \mathrm{c}-\mathrm{g}$ & 18.7a-h & $74.1 \mathrm{c}-\mathrm{g}$ \\
\hline 29 & SB26 & $77 a-e$ & 2044ab & $3.32 \mathrm{ij}$ & $9.42 \mathrm{abc}$ & $10.5 \mathrm{c}-\mathrm{g}$ & $12.6 \mathrm{i}$ & $74.2 \mathrm{c}-\mathrm{g}$ \\
\hline 30 & SB8 & $73 a-g$ & $1831 b-e$ & $4.22 \mathrm{a}-\mathrm{e}$ & $7.21 \mathrm{a}-\mathrm{f}$ & $10.9 b-g$ & 18.6a-g & $80.9 b-g$ \\
\hline 31 & SB22 & $69 a-h$ & $1777 b-e$ & $3.62 d-j$ & $6.59 \mathrm{edf}$ & $9.4 \mathrm{fg}$ & 13.3h-i & 67.9efg \\
\hline 32 & SB38 & $83 \mathrm{ab}$ & $1817 b-e$ & $3.65 \mathrm{~d}-\mathrm{j}$ & $7.72 \mathrm{a}-\mathrm{f}$ & $11.9 \mathrm{a}-\mathrm{f}$ & $16.6 \mathrm{~d}-\mathrm{i}$ & $77.1 \mathrm{c}-\mathrm{g}$ \\
\hline 33 & SB36 & $75 a-f$ & $1625 b-e$ & 3.34hij & $8.45 a-d$ & $11.1 \mathrm{~b}-\mathrm{g}$ & $17.8 \mathrm{a}-\mathrm{i}$ & $64.4 \mathrm{~g}$ \\
\hline 34 & SB7 & $83 a b$ & $1785 b-e$ & $3.89 \mathrm{~b}-\mathrm{i}$ & 7.56a-f & $11.6 \mathrm{a}-\mathrm{g}$ & $16.9 b-i$ & $75.1 \mathrm{c}-\mathrm{g}$ \\
\hline 35 & SB9 & $79 a-d$ & $1922 a-c$ & $4.33 \mathrm{a}-\mathrm{d}$ & $8.03 a-f$ & $13.5 \mathrm{ab}$ & $18.4 \mathrm{a}-\mathrm{g}$ & $80.3 b-g$ \\
\hline 36 & SB34 & $73 a-e$ & $1858 b-e$ & $3.81 \mathrm{~b}-\mathrm{j}$ & 8.83a-d & $11.1 \mathrm{~b}-\mathrm{g}$ & $19.7 \mathrm{a}-\mathrm{g}$ & $70.5 \mathrm{~d}-\mathrm{g}$ \\
\hline 37 & SB29 & $73 a-e$ & $1913 a-d$ & $3.37 \mathrm{~h}-\mathrm{i}$ & $7.70 \mathrm{a}-\mathrm{f}$ & $12.7 \mathrm{a}-\mathrm{d}$ & $20.2 a-f$ & $73.7 \mathrm{c}-\mathrm{g}$ \\
\hline 38 & Sepid & $77 a-e$ & $1522 \mathrm{c}-\mathrm{e}$ & 3.91a-i & $7.03 a-f$ & $11.5 \mathrm{a}-\mathrm{g}$ & $18.3 \mathrm{a}-\mathrm{g}$ & $77.1 \mathrm{c}-\mathrm{g}$ \\
\hline 39 & SB33 & $87 a$ & $1949 a-c$ & $4.02 \mathrm{a}-\mathrm{i}$ & $7.58 \mathrm{a}-\mathrm{f}$ & $11.5 \mathrm{a}-\mathrm{g}$ & $15.8 \mathrm{e}-\mathrm{i}$ & $70.8 \mathrm{~d}-\mathrm{g}$ \\
\hline 40 & Golestan & $81 \mathrm{abc}$ & $2116 a b$ & $4.06 \mathrm{a}-\mathrm{g}$ & $9.51 \mathrm{ab}$ & $11.4 \mathrm{a}-\mathrm{g}$ & $13.9 \mathrm{~g}-\mathrm{i}$ & $65.1 \mathrm{~g}$ \\
\hline
\end{tabular}




\section{Stability analysis}

Based on how to do statistical analysis, there are a lot of methods to evaluate the genotype $\times$ environment interaction that usually divided it into two major groups of parametric and nonparametric. Versus genotypes and environment interaction, the stability subject is raised. Term stability is used about genotypes that have a relatively stable performance and the minimal variance in different environmental conditions. This aspect of stability called static or biological stability. Of course, such genotype is not always the best genotype because not responds to improve environmental conditions.

\section{Romer environmental variance}

Phenotypic stability according to the concept of static stability (stable) by Romer (10) using variance of a genotype in different environments is calculated and measures deviation of a genotype from average of that genotype in all environments. A genotype that not responds to changing environmental conditions is more stable. The standard is an independent model of other genotypes. According to environmental variance, a genotype is stable that its environmental variance is less. Of course, this stability should not be generalized i.e. a genotype that with a group of genotypes seems stable may be unstable with another group of genotypes. Based on this, according to the results in Table 4 and 5, genotype 1 which has the lowest yield, also has had the lowest environmental variance and is considered as the most stable genotype. In contrast, genotype 29 that was part of yielding genotypes has had the highest variance and from this point is accounted as an unstable genotype.

Table 4. Average cotton seed yield of 40 genotypes in different environments $(\mathrm{Kg} / \mathrm{ha})$

\begin{tabular}{|c|c|c|c|c|c|c|c|}
\hline A & $\mathrm{y} 1 * \mathrm{~L} 1$ & $\mathrm{y} 1 * \mathrm{~L} 2$ & $\mathrm{y} 1 * \mathrm{~L} 3$ & $\mathrm{y} 2 * \mathrm{~L} 1$ & $\mathrm{y} 2 * \mathrm{~L} 2$ & $\mathrm{y} 2 * \mathrm{~L} 3$ & mean \\
\hline 1 & 1729 & 1109 & 1479 & 2010 & 831 & 1183 & 1390.1 \\
\hline 2 & 2368 & 967 & 1833 & 2438 & 867 & 1467 & 1656.7 \\
\hline 3 & 2682 & 1257 & 1642 & 1174 & 934 & 1314 & 1500.6 \\
\hline 4 & 2396 & 993 & 1626 & 1272 & 1039 & 1301 & 1437.7 \\
\hline 5 & 2657 & 698 & 1708 & 2484 & 944 & 1366 & 1642.9 \\
\hline 6 & 3195 & 857 & 1833 & 1973 & 946 & 1466 & 1711.5 \\
\hline 7 & 2715 & 824 & 1733 & 984 & 872 & 1386 & 1418.9 \\
\hline 8 & 3141 & 1267 & 2252 & 2105 & 1001 & 1801 & 1927.8 \\
\hline 9 & 2610 & 1396 & 1781 & 1644 & 1015 & 1424 & 1645.0 \\
\hline 10 & 1919 & 704 & 1518 & 2773 & 807 & 1214 & 1489.0 \\
\hline 11 & 2388 & 621 & 2183 & 1342 & 897 & 1747 & 1529.6 \\
\hline 12 & 3157 & 729 & 2009 & 2566 & 1062 & 1607 & 1855.0 \\
\hline 13 & 2297 & 626 & 2414 & 1927 & 800 & 1931 & 1665.8 \\
\hline 14 & 2499 & 976 & 1620 & 1625 & 869 & 1296 & 1480.7 \\
\hline 15 & 3333 & 1005 & 2421 & 1688 & 976 & 1937 & 1893.2 \\
\hline 16 & 2968 & 1441 & 2030 & 2059 & 932 & 1624 & 1842.3 \\
\hline 17 & 3389 & 1091 & 2129 & 1748 & 867 & 1703 & 1821.4 \\
\hline 18 & 3663 & 1522 & 2924 & 2725 & 947 & 2340 & 2353.4 \\
\hline 19 & 3181 & 1400 & 2635 & 2098 & 924 & 2108 & 2057.8 \\
\hline 20 & 3505 & 1844 & 2128 & 2865 & 995 & 1703 & 2173.4 \\
\hline 21 & 3219 & 880 & 1601 & 1906 & 1248 & 1281 & 1689.3 \\
\hline 22 & 3102 & 1188 & 2446 & 2169 & 1082 & 1957 & 1990.8 \\
\hline 23 & 3008 & 1682 & 2461 & 2323 & 985 & 1969 & 2071.4 \\
\hline 24 & 3371 & 2184 & 2502 & 2199 & 1162 & 2002 & 2236.8 \\
\hline 25 & 3289 & 1332 & 1471 & 2532 & 1086 & 1176 & 1814.3 \\
\hline 26 & 3095 & 1321 & 2439 & 1734 & 906 & 1951 & 1907.8 \\
\hline 27 & 3278 & 821 & 2813 & 1975 & 1153 & 2250 & 2048.2 \\
\hline 28 & 3071 & 1345 & 2082 & 2111 & 893 & 1666 & 1861.2 \\
\hline 29 & 3985 & 1068 & 2132 & 2980 & 1196 & 1705 & 2177.7 \\
\hline 30 & 3093 & 1727 & 2608 & 1317 & 961 & 2087 & 1965.3 \\
\hline 31 & 3236 & 1599 & 1993 & 2113 & 932 & 1594 & 1911.1 \\
\hline 32 & 3714 & 1207 & 1961 & 2073 & 1179 & 1569 & 1950.5 \\
\hline 33 & 2900 & 1073 & 2727 & 776 & 897 & 2182 & 1759.2 \\
\hline 34 & 3415 & 1081 & 2382 & 1737 & 994 & 1906 & 1919.1 \\
\hline 35 & 3321 & 987 & 2422 & 2522 & 1149 & 1937 & 2056.2 \\
\hline 36 & 2941 & 2173 & 2441 & 1544 & 899 & 1953 & 1991.8 \\
\hline 37 & 3638 & 803 & 2514 & 2463 & 850 & 2011 & 2046.5 \\
\hline 38 & 2720 & 887 & 2462 & 915 & 983 & 1970 & 1656.2 \\
\hline 39 & 3097 & 1423 & 2725 & 1936 & 1135 & 2180 & 2082.7 \\
\hline 40 & 3276 & 1216 & 2708 & 2474 & 1657 & 2167 & 2249.8 \\
\hline mean & 3219 & 880 & 1601 & 1906 & 1248 & 1281 & 1846.981 \\
\hline
\end{tabular}


SADABADI M. FATHI, et al.

Table 5. Calculation of performance stability parameters of cotton genotypes in different environments

\begin{tabular}{|c|c|c|c|c|c|c|c|c|}
\hline A & mean & $S^{2}$ & $\mathrm{Cv}$ & $\mathrm{Wi}^{2}$ & $\delta^{2}$ & $\mathrm{~b}$ & $S^{2} d$ & $\mathrm{R}^{2}$ \\
\hline 1 & 1390.1 & 188477.5 & 31.2 & 1216037.0 & 252844.2 & 0.4485 & 101847.8 & 0.5677 \\
\hline 2 & 1656.7 & 456546.8 & 40.8 & 656439.9 & 135034.3 & 0.8055 & 139134.7 & 0.7562 \\
\hline 3 & 1500.6 & 387651.8 & 41.5 & 509898.9 & 104183.6 & 0.7685 & 91763.1 & 0.8106 \\
\hline 4 & 1437.7 & 271085.7 & 36.2 & 405574.2 & 82220.5 & 0.6786 & 32608.4 & 0.9038 \\
\hline 5 & 1642.9 & 639907.1 & 48.7 & 717304.1 & 147847.8 & 0.9664 & 178765.4 & 0.7765 \\
\hline 6 & 1711.5 & 731901.2 & 50.0 & 217944.6 & 42719.5 & 1.1468 & 40206.2 & 0.9561 \\
\hline 7 & 1418.9 & 524193.6 & 51.0 & 444974.7 & 90515.3 & 0.9091 & 105580.6 & 0.8389 \\
\hline 8 & 1927.8 & 583925.3 & 39.6 & 9927.6 & -1073.6 & 1.0469 & 1040.4 & 0.9986 \\
\hline 9 & 1645.0 & 291407.8 & 32.8 & 326694.1 & 65614.1 & 0.7125 & 26692.0 & 0.9267 \\
\hline 10 & 1489.0 & 598130.3 & 51.9 & 2018787.7 & 421844.4 & 0.6824 & 437934.4 & 0.4143 \\
\hline 11 & 1529.6 & 494741.5 & 46.0 & 523924.7 & 107136.4 & 0.8666 & 118948.2 & 0.8077 \\
\hline 12 & 1855.0 & 836628.0 & 49.3 & 613826.3 & 126063.0 & 1.1708 & 134206.2 & 0.8717 \\
\hline 13 & 1665.8 & 585538.5 & 45.9 & 767081.8 & 158327.3 & 0.9062 & 185815.8 & 0.7461 \\
\hline 14 & 1480.7 & 348228.4 & 39.9 & 143383.8 & 27022.5 & 0.8003 & 9342.7 & 0.9785 \\
\hline 15 & 1893.2 & 805037.6 & 47.4 & 310881.7 & 62285.2 & 1.1982 & 51454.8 & 0.9489 \\
\hline 16 & 1842.3 & 477571.4 & 37.5 & 110444.1 & 20087.8 & 0.9280 & 24236.9 & 0.9594 \\
\hline 17 & 1821.4 & 803053.6 & 49.2 & 219610.6 & 43070.2 & 1.2135 & 24566.8 & 0.9755 \\
\hline 18 & 2353.4 & 969613.5 & 41.8 & 484436.4 & 98823.0 & 1.3201 & 53012.4 & 0.9563 \\
\hline 19 & 2057.8 & 663462.7 & 39.6 & 182494.5 & 35256.3 & 1.0893 & 40278.8 & 0.9514 \\
\hline 20 & 2173.4 & 794814.1 & 41.0 & 822682.7 & 170032.8 & 1.0923 & 200001.3 & 0.7987 \\
\hline 21 & 1689.3 & 682122.6 & 48.9 & 583843.2 & 119750.8 & 1.0313 & 145308.6 & 0.8296 \\
\hline 22 & 1990.8 & 588991.8 & 38.5 & 51273.6 & 7630.9 & 1.0439 & 11537.1 & 0.9843 \\
\hline 23 & 2071.4 & 486933.3 & 33.7 & 202940.5 & 39560.7 & 0.9195 & 46422.5 & 0.9237 \\
\hline 24 & 2236.8 & 514603.9 & 32.1 & 474311.1 & 96691.4 & 0.8945 & 111171.6 & 0.8272 \\
\hline 25 & 1814.3 & 795396.2 & 49.2 & 1203870.4 & 250282.8 & 1.0212 & 300667.9 & 0.6976 \\
\hline 26 & 1907.8 & 614165.3 & 41.1 & 192102.5 & 37279.0 & 1.0411 & 46903.3 & 0.9389 \\
\hline 27 & 2048.2 & 890203.4 & 46.1 & 659761.9 & 135733.7 & 1.2126 & 134881.8 & 0.8788 \\
\hline 28 & 1861.2 & 563176.2 & 40.3 & 68464.2 & 11249.9 & 1.0164 & 16937.2 & 0.9759 \\
\hline 29 & 2177.7 & 1266050.8 & 51.7 & 1337823.0 & 278483.4 & 1.4384 & 206655.9 & 0.8694 \\
\hline 30 & 1965.3 & 637434.5 & 40.6 & 976795.3 & 202477.5 & 0.9155 & 239445.7 & 0.6995 \\
\hline 31 & 1911.1 & 591906.1 & 40.3 & 270114.2 & 53702.6 & 1.0055 & 67508.5 & 0.9088 \\
\hline 32 & 1950.5 & 884201.5 & 48.2 & 539337.5 & 110381.1 & 1.2296 & 99778.1 & 0.9097 \\
\hline 33 & 1759.2 & 919276.4 & 54.5 & 1952056.7 & 407795.7 & 0.9970 & 488008.3 & 0.5753 \\
\hline 34 & 1919.1 & 808698.2 & 46.9 & 273970.8 & 54514.5 & 1.2085 & 39578.1 & 0.9608 \\
\hline 35 & 2056.2 & 786142.4 & 43.1 & 288497.8 & 57572.8 & 1.1846 & 49465.3 & 0.9497 \\
\hline 36 & 1991.8 & 506337.4 & 35.7 & 1181872.4 & 245651.7 & 0.7537 & 255127.4 & 0.5969 \\
\hline 37 & 2046.5 & 1180705.3 & 53.1 & 741773.8 & 152999.3 & 1.4702 & 38413.6 & 0.9740 \\
\hline 38 & 1656.2 & 694661.8 & 50.3 & 1233637.9 & 256549.7 & 0.9210 & 304255.3 & 0.6496 \\
\hline 39 & 2082.7 & 561118.4 & 36.0 & 257557.8 & 51059.1 & 0.9789 & 64094.1 & 0.9086 \\
\hline 40 & 2249.8 & 547962.7 & 32.9 & 249791.6 & 49424.1 & 0.9680 & 61767.8 & 0.9098 \\
\hline
\end{tabular}

\begin{abstract}
Coefficient of genotypic variations of Francis and Kanenberg

Francis and Kanenberg (11) to determine the stability of corn varieties used the coefficient of variations of a genotype that measures the share of genotype I in the interaction between genotype and environment and is independent from the other tested genotypes. In fact, $\mathrm{CVi}$ measures deviation of a genotype from average genotypes in all environments. According to this criterion, a genotype is more stable when genotype variation coefficient is less. As it is shown in Table 5, genotype 1 was the most stable genotype but genotype 33 has more coefficient of variation and was more unstable than the other tested genotypes.
\end{abstract}

\section{Wricke equivalence}

Wricke (12) suggested that the interaction of genotype and environment for each genotype can be used as a parameter of stability, as this effect be squared for any genotype and added up in all environments. Genotypes that have small equivalence have less volatility and they are more stable. This parameter is directly related to the genotype $\times$ environment interaction and its calculation is simple. Considering that equivalence measures the contribution of each genotype in the interaction. Thus, a genotype with $\mathrm{Wi}^{2}=0$ is considered as stable. According to data in Table 5, genotype 8 has had more stability but genotypes 29, 33 and 10 were the most unstable genotypes. 


\section{Shukla stability variance}

Shukla (13) proposed variance estimate of genotype $\mathrm{i}$ in different environments based on remainder of the mutual classification of genotype and environment. Stability variance is linear combinations of equivalence so rating genotypes were similar in both models. Because the stability variance is based on the remaining of matrix of interaction of genotype and environment, so the more the variance, indicates less the main effect of genotype is considering as more stable genotype. Ranking of genotypes based on Shukla stability variance is somewhat similar to Rick equivalence. Thus, the most stable genotype was genotype number 8 . However, genotypes 29,33 and 10 were the most unstable genotypes.

\section{Finlay and Wilkinson}

In this method, two parameters of regression coefficient and deviation from regression as stable parameters are used and regression of genotype and environment interaction on environmental index is calculated, the difference is that before calculating the regression coefficient not needs to correct the environmental effects on values. Also, the performance of genotypes as main effects for genotype and environment and product of main effects of environment in regression coefficients is expressed. With this parameter, a genotype that has a gradient of $1(b=1)$ has the highest stability than the other genotypes. Finlay and Wilkinson (14) demonstrated that bi parameter in addition to stability represents compability. Genotypes that significantly have regression coefficient greater than one have special compatibility for environments with high performance. However, according to the results in Table 5 genotype 33 with $b=0.997$ was the most stable genotype and genotypes number 29 and 37 were more unstable than the other genotypes and also genotype 29 is suitable for environments with good performance like Hashemabad.

\section{Eberhart and Russell}

Eberhart and Russell (15) measured varieties respond to environmental changes with coefficient of linear regression and deviations from the regression and grouped varieties based on regression coefficient less or more than one and variance of deviation equal to or different from zero. In this method the linear regression coefficient is accounted as the compatibility parameter and the deviation from regression is considered as the parameter of stability and nonlinear component. If the regression coefficient has not a significant difference with one, it should be benefitted as the deviation of regression and average of yield because yield is associated with the linear response of varieties. This analysis is useful only if at least $50 \%$ of total sum of squares is expressed by sum of squares of interaction between genotype and environment. According to Eberhart and Russell (15), a genotype is stable when mean of squares of deviation is smaller than its regression. In Table 5, this specification is shown by genotype 8 while in genotype 33 the regression coefficient was near one but its sum of squares for regression deviation was higher than the other genotypes.

\section{Determination Coefficient of Pintus}

Pintus (16) proposed determination coefficient instead of mean of squares for regression deviation to be used in calculating the genotype stability. This parameter strongly depends on the studied genotypes and deviation from regression. A genotype with more coefficient of determination is more stable. Basically, genotype 8 showed a good stability, however genotypes 10 and 33 were unstable. Finally, after calculating the stability parameters and according to regression slope near one, it can be introduced that genotype 8 as the most stable genotype among all 40 studied genotypes since it has smaller share in the interaction of genotype and environment according to Wricke (12) ecovalans parameter and Shukla (13) stability variance. Genotypes 10 and 33 were the most unstable genotypes in terms of performance.

\section{Determining the compatibility and yield stability}

Determining the compatibility and yield stability is one of the most important and costly stages in reform program of crops. This issue is important because of the genotype interaction on the environment simply is not possible. The genotype interaction on the environment will be important when the interaction is significant and various genotypes show their superiority in different environments. The performance of a genotype in each environment consisting of the main effect of environment (E), the main effect of genotype $(\mathrm{G})$, and the genotype $\times$ environment (GE) interaction. Yan (17) stated that despite the effect of environment allocated a major role from changes of the whole performance and the effects of genotype and genotype $\times$ environment interaction are small but these two effects were involved in assessment tests of genotypes and at time of selection of superior genotypes, genotype effect and genotype interaction on the environment must be considered simultaneously. Three methods 
of statistical analysis include principal components analysis (PCA), additive main effects and multiplicative interaction (AMMI) and GGE biplot analysis based on singular value decomposition (SVD) are established and often used for the analysis of experimental data comparing the performance in area.

\section{Analysis in AMMI method}

AMMI method justifies the main part of the sum of squares of genotype $\times$ environment interaction. In contrast to the regression methods that is not able to predict the nonlinear of response of genotypes to the environment, separate main and interaction effects and estimates performance. Also, it makes possible interpretation of analyzed data. This model uses incremental analysis for the main effects and multiplicative analysis for interactions and is a combination of variance analysis and analysis to principal component.

AMMI model reduces errors and its statistical benefit is equal to add 1 to 3 replications in a testing experiment. IPCA is stability score of genotype that scores more negative or positive indicates compatibility to a particular environment and scores close to zero means greater stability. It may be obtained a large number of IPC in analysis of AMMI but much IPC causes error and two IPC seem to be enough for analysis.

In Figure 2, diagram of analysis by AMMI method based on two components IPC1, IPC2 is shown. Share of the first component explained 49\%, the second component explained $22 \%$ and overall they explained $71 \%$ of the variance. The first group that have IPC1 positive are appropriate for Hashemabad region, includes genotypes TJ82, SB29, Sb9, SB26 and Golestan commercial cultivars which are high yielding varieties. The second group includes genotypes DB19, DB22, and SB34, which are unstable cultivars and are suitable for Anbarolum region. The third group includes genotypes SB36, TJ8, TJ185 that with the commercial cultivars of Sepid has special are compatible to Gonbad region.

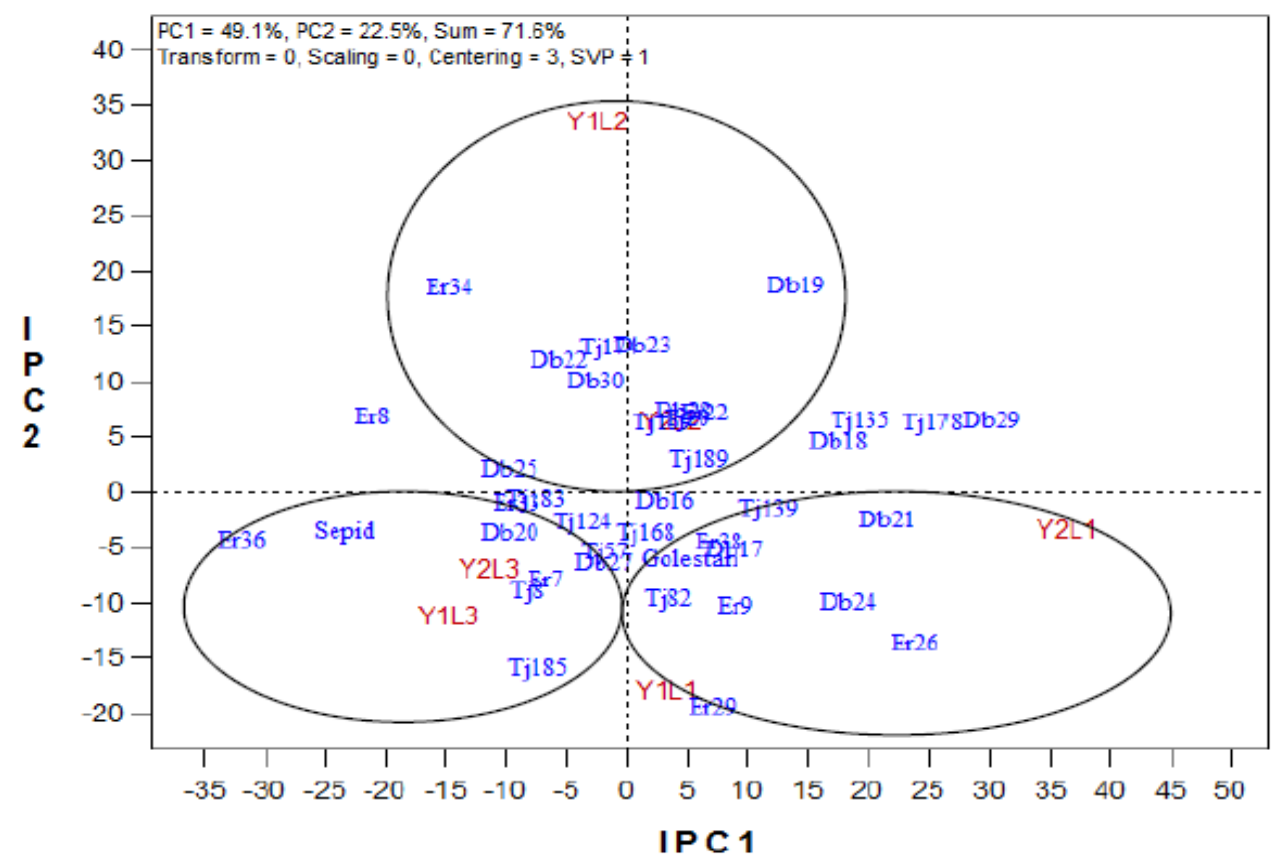

GGE biplot

Figure 2. Diagram of analysis by the AMMI method based on two components

\section{Biplot analysis}

Biplot technique was first proposed by Gabriel (18) and then was developed by Kempton (19) and Zobl et al (20), but its widespread use in examining the genotype $(\mathrm{G})$ and genotype $\times$ environment (GE) recently (21). In the GGE biplot main effects of genotype (G) and genotype $\times$ environment (GE) are not separated from each other. Base on view of breeders and producers genotypes selection on $\mathrm{G}$ or $\mathrm{GE}$ crops alone is not considered. So study these effects have to be done simultaneously. That's why Yan et al (17) believe that GGE biplot analysis is more successful than genotypic data in the AMMI method.

There are many ways to characterize the interaction of $\mathrm{G} \times \mathrm{E}$. Charts that simultaneously show genotypes reaction in different environments (biplot) have much important and wide uses. GGE biplot is a tool 
for data visualization because shows interaction between genotype and environment in a bilateral graph. Hens, to determine the stability of various products such as rice (22), barley (23), wheat (24) and lentils (25) have been used.

To identify superior genotypes and environments, polygon graph was plotted by software GGE biplot. The corresponding polygon is obtained from connection of genotypes that have the maximum distance from the origin. On this basis, superior genotypes included TJ82, ER26, DB29, DB19, DB25, and ER36 that are placed at the head of the polygon. Using vertical lines on the sides of the polygon, larger environments were identified that in this figure, Hashemabad environment placed in one group and Anbarolum and Gonbad regions placed in another group. Another consequence is that because of placing genotype ER26 in top of the first group of environments is suitable for Hashemabad and genotype TJ82 is appropriate for second group of areas. Other genotypes were not placed in any environment, which indicates that these genotypes showed no superiority in none of environments. Of course, two components specified in this figure justify totally $74 \%$ of the variations (Figure 3).

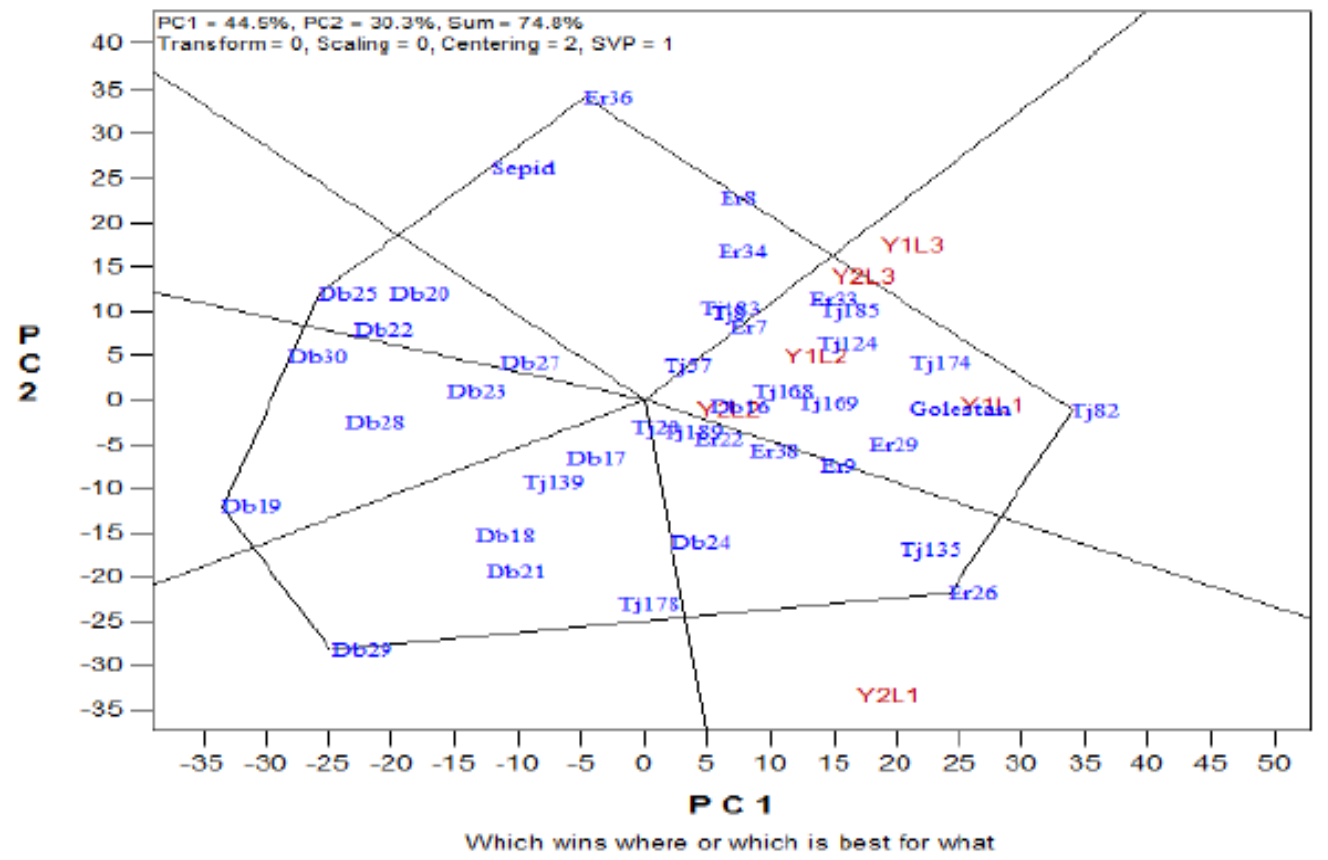

Figure 3. Graphical representation of compliance of 40 cotton genotypes with three regions Hashemabad, Anbarolum and Gonbad in two years (2015-2014)

If the sum of the first and second principal component fails most of the variation, that reflects the complex nature of the interaction between genotype environment and biplot not necessarily mean invalid.

In Figure 4, the average of performance of genotypes and their stability is simultaneously studied. Genotypes that are placed in positive side of $\mathrm{X}$ axis i.e. genotype TJ82 had the highest performance and in contrast, genotypes in left side of X axis, such as DB19 had the lowest performance. Genotypes ER36, DB29 in addition to low yield, had the most instability. Because the more the length of the perpendicular line on the horizontal axis is more, it represents the instability of the genotype. Finally, genotype TJ82 was selected as the best and most stable while Golestan cultivar also had a good performance and stability (Figure 4).

Survey results showed that the GGE biplot an easy way to evaluate the effect of genotype on the environment and useful information about the genotypes and environments provides under review. Other researchers have reported similar results (5-26-27).

\section{Abbreviation}

AMMI: Additive main effects and multiplicative interaction;

GEI: Genotype-environment interaction;

GGE: Genotype main effects and genotype $\times$ environment interaction effects;

AEC: average environment coordinate;

PCA: Principal components analysis;

SVD: Singular value decomposition;

IPCA: interaction principal component axis; HVI: High Volume Instrumentation. 


\section{ACKNOWLEDGMENTS:}

I would like to express appreciation to my advisor Dr. Ranjbar and Dr. Zangi, who's theirs, guidance in my professional and personal life will last a lifetime. I would also like to thank Dr. Roshani, head of Research Cotton Institute of Iran for providing financial support during the course of this research.

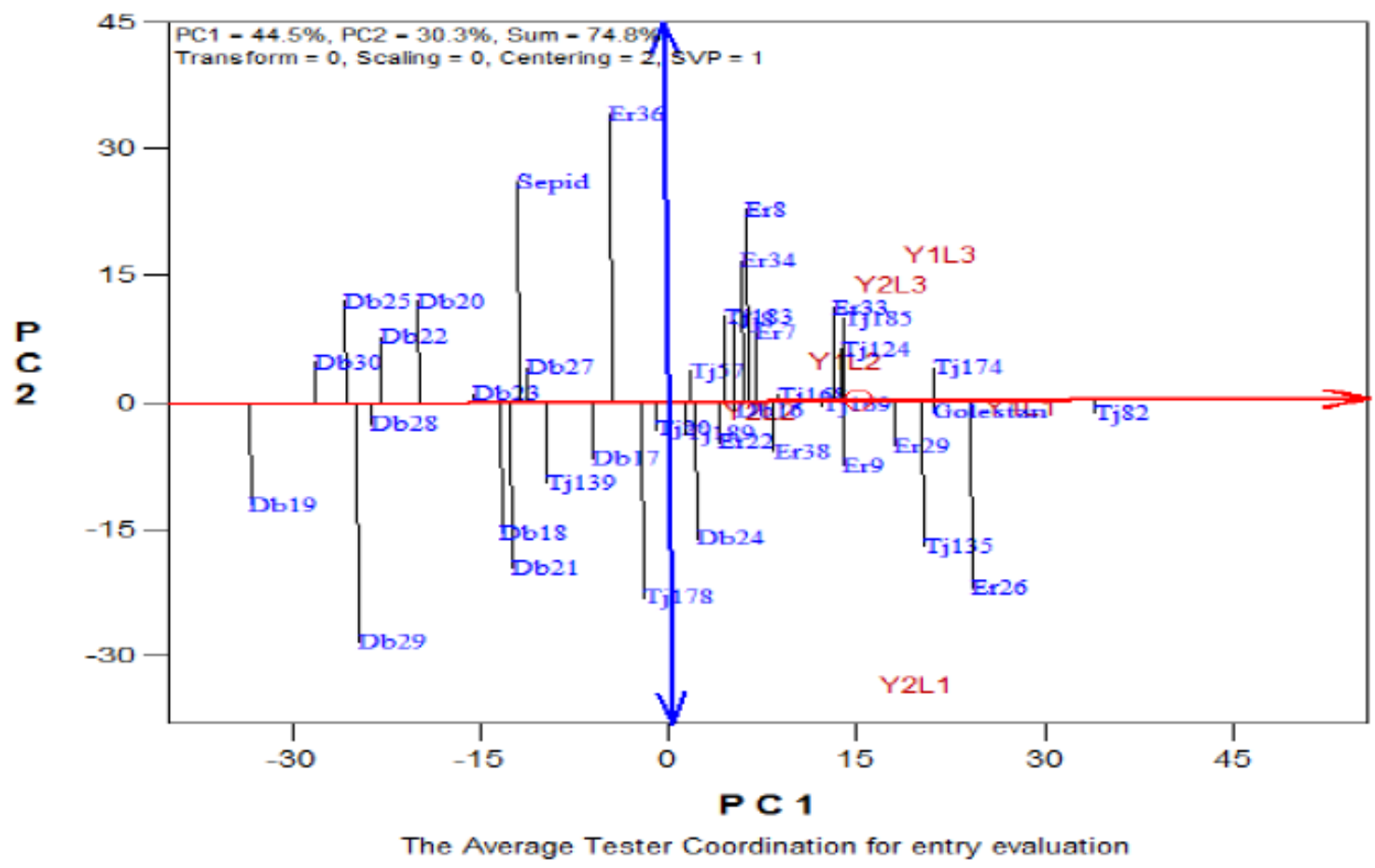

Figure 4. Evaluate cotton genotypes in 6 environments based on yield and stability simultaneously

\section{REFRENCES}

1. Singh, J. Final report of Impact Assessment IPM Cotton project for boosting diversification process in Punjab. 2011. https://www.researchgate.net/publication/2 66082386

2. Alishah, O., Study on adaptability and quantitative and qualitative characteristics of hopeful cotton cultivars in mild and cold regions of Iran. Final Report Cotton Research Institute of Iran, 34 p. 2014. (in Persian).

3. Soughi, H. Kalateh, M. Abroudy, SA., Stability analysis of grain yield and inter relationships of characters of bread wheat advanced lines in Gorgan. Pajouhes \& Sazandegi 70: 56-62. 2006. (in Persian).

4. Damavandi Kamali, S. Babaeian Jelodar, N. Alishah, O., The Assessment of Adaptability and Stability of Yield on Cotton Cultivars by Using Uniparametric, Non-Parametric Methods and AMMI Mode. IRANIAN JOURNAL OF FIELD CROP SCIENCE. 42-2: 397-407.2011. (in Persian).

5. Pourdad, SS. Jamshidi Moghaddam, M., Study on Genotype $\times$ Environment Interaction Through GGE Biplot for Seed Yield in Spring Rapeseed (Brassica Napus
L.) in Rain-Fed Condition. Journal of Crop Breeding Vol. 5, No. 12. $2013 . \quad$ (in Persian).

6. Lalbachan, V. Analysis of genotypeenvironment interaction for yield in irrigated rice. College Laguna j. Philipin. 1994.

7. Li, G. Xiao, H. Fang, H., Analysis on stability of the main economic characters of flue-cured tobacco cultivars. Acta Tabacaria Sinica, 2001, 7-1, p. 13-8. 2001.

8. Campbell, BT. Jones, MA., Assessment of genotype $\times$ environment interactions for yield and fiber quality in cotton performance trials. Euphytica 144: 69-78. 2005.

9. Farshadfar, E. Mahmodi, N. Yaghotipoor, A., AMMI stability value and simultaneous estimation of yield and yield stability in bread wheat (Triticum aestivum L.). Aust J Crop Sci 5(13):1837-1844. 2011.

10.Rommer, TH. Sind die ertragreicheren sorten ertragssicherer? DGL-Kitt 32: 87-89. 1947.

11.Francis, TR. Kanenberg, LW., Yield stability studies in short season. Maize.I.A descriptive method for grouping genotypes Can. J. Plant sci.58:1024-1034. 1987. 
12.Wricke, G., Über eine Methode zur Erfassung der Okasiagischen Streubreite in Feld Versuchen. Pflanzuecht 47: 92-96. 1962.

13.Shukla, GK., Some statistical aspects of partitioning genotype - environmental components of variability. Heredity, 29, 237-245. 1972.

14. Finlay, KW. Wilkinson, GN., The analysis of adaptation in a plant breeding program. Australian Journal of Agricultural Research 14: 742-754. 1963.

15. Eberhart, SA. Russell, WA., Stability parameters for comparing varieties. Crop Science 6: 36-40. 1966.

16. Pinthus, M. J., Estimate of genotype value: A personal method. Euphytica, 22, 121123. 1973.

17.Yan, W. Kang, MS. Woods BMa, S. Cornelius, PL., GGE biplot vs. AMMI analysis of genotype -by- environment data. Crop Science, 47: 643-655. 2007.

18. Gabriel, K R. The biplot graphic display of matrices with application to principal component analysis. Biometrika 58: 453467. 1971.

19.Kempton, RA. The use of biplots in interpreting variety by environment interaction. Agricultural cientific, 103, 123135. 1984.

20.Zobel, RW. Wright, MJ. Gauch, HG., Statistical analysis of a yield trial, Agronomy Journal, 80: 388-393. 1988.
21.Yan, W. Kang, MS., GGE biplot analysis: A graphical tool for breeders, Geneticists and agronomists. CRC Press, Boca Raton, FL. 2003.

22.Samonte, SOPB. Wilson, LT. Mc Clung, AM. Medley, JC., Targeting cultivars onto rice growing environments using AMMI and SREG GGE biplot analysis. Crop Science, 45: 2414-2424. 2005.

23. Dehghani, H. Ebadi, A. Yousefi, A., Biplot analysis of genotype by environment interaction for Barley yield in Iran. Agronomy Journal, 98: 388-393. 2006.

24. Kaya, Y. Akcura, M. Taner, S., GGE biplot analysis of multi-environment yield trials in bread wheat. Turkish Journal Agriculture Forestry, 30: 325-337. 2006.

25. Sabaghnia, N. Dehghani, H. Sabaghpour, SH., Graphic Analysis of Genotype by Environment Interaction for Lentil Yield in Iran. Agronomy Journal, 100: 760-764. 2008.

26.Kamali, A. Fakheri, BA. Zabet, M., The study of drought stress effects on yield and yield components of cotton using biplot analysis. Iranian Journal of Cotton Researches Vol. 3, No. 1.2015. (in Persian). 27.Jamshid Moghaddam, M. Pourdad, SS., Genotype $\times$ environment interactions for seed yield in rainfed winter safflower (Carthamus tinctorius L.) multienvironment trials in Iran. Euphytica 190:357-369. 2013 